\title{
Comparative Evaluation of Gene Xpert and Various Staining Techniques in the Diagnosis of Pulmonary Tuberculosis
}

\author{
Loveena Oberoi $^{1}$, Naveen Pandhi ${ }^{2}$, Muskan Khullar ${ }^{1 *}$ and Tavishi Oberoi ${ }^{1}$ \\ ${ }^{1}$ Department of Microbiology, ${ }^{2}$ Department of Chest and TB, Government Medical College, \\ Amritsar, India \\ *Corresponding author
}

\section{Keywords}

Ziehl Neelsen (ZN) staining, Kinyoun

staining,

Fluorescent

staining,

Tuberculosis (TB),

Mycobacterium

tuberculosis

(MTB), N-acetyl-Lcysteine (NALC)

Article Info

Accepted:

20 May 2021

Available Online:

10 June 2021
A B S T R A C T

Tuberculosis (TB) is an ancient communicable disease caused by Mycobacterium tuberculosis. In India, RNTCP (Revised national tuberculosis control program) now known as NTEP (National tuberculosis elimination program) endorsed diagnostic steps to diagnose TB infection and disease are followed. To study the diagnostic efficacy of GeneXpert in comparison to various staining techniques in patients suspected of pulmonary tuberculosis. 150 sputum samples from patients clinically suspected of pulmonary tuberculosis were processed and subjected to Ziehl-Neelsen staining, Kinyoun Staining and Fluorescent staining. Simultaneously GeneXpert was performed on unprocessed samples. Out of 150 samples, GeneXpert detected $46(30.67 \%)$ cases followed by Fluorescent staining, 44(29.33\%), ZN staining $43(28.67 \%)$ cases and Kinyoun staining detected the least i.e. $35(23.33 \%)$. Amongst 46 positive cases detected by GeneXpert $7(15.22 \%)$ cases were found to be resistant to Rifampicin. Hence it proved the efficacy and sensitivity of GeneXpert over staining methods. Tuberculosis is a global pandemic. Its early diagnosis and timely initiation of treatment is necessary. Therefore, GeneXpert when used in conjunction with microscopy improves detection of tuberculosis and multi drug resistant tuberculosis.

\section{Introduction}

Tuberculosis (TB) is potentially infectious disease caused by Mycobacterium tuberculosis, which can affect any part of the body with the lungs being the most common organ involved. ${ }^{1}$ The World Health Organization (WHO) estimates that 1.8 billion people-close to one-quarter of the world's population-are infected with this deadly disease. ${ }^{2}$ In the Indian scenario, the estimated TB incidence is about 27 lakh with the majority of the affected individuals being in the age group of $15-69$ years and $2 / 3^{\text {rd }}$ being males. ${ }^{3}$ Rapid and accurate diagnosis of pulmonary tuberculosis followed by prompt treatment with effective anti-TB drugs is the cornerstone of TB management and 
elimination of transmission as endorsed by RNTCP, now known as NTEP.

Sputum smear microscopy by Ziehl-Neelsen staining is considered as the most common diagnostic test available. But nowadays, Fluorescent microscopy is being preferred over conventional light microscopy as it is more efficient and can help in diagnosing TB at the earliest. ${ }^{4}$ Another potential alternative to ZN Staining is Kinyoun's Cold Acid Fast staining which differs from former as in that case heating is not required, phenol concentration in carbol fuchsin is increased and duration of carbol fuchsin staining is more. ${ }^{5}$ The only demerit of microscopy is low sensitivity $(50-80 \%)$ relative to culture. The sensitivity of microscopy can be improved by prior sputum decontamination methods by NAcetyl-L-Cysteine Sodium Hydroxide (NALC-NaOH) method and bleach method using $5 \%$ Sodium Hypochlorite. ${ }^{6}$

Newer molecular techniques like GeneXpert $\mathrm{MTB} / \mathrm{RIF}$ assay is another diagnostic platform which is automated, cartridge based, closed system that performs real-time PCR and can be used by operators with minimal technical expertise, enabling diagnosis of $\mathrm{TB}$ and simultaneous assessment of rifampicin resistance to be completed within 2 hours leading to timely initiation of treatment with minimal biohazard. ${ }^{7,8}$

The main objectives of this study to evaluate efficacy of GeneXpert and comparative evaluation of GeneXpert with various staining techniques in the suspected cases of pulmonary tuberculosis.

\section{Materials and Methods}

The present cross sectional study was performed in Microbiology Department in collaboration with Chest \& TB hospital and other wards of Guru Nanak Dev Hospital,
Amritsar. A total of 150 Sputum samples from Patients with clinical suspicion of pulmonary tuberculosis including symptoms of cough with or without expectoration for $>2$ weeks, weight loss, fatigue, hemoptysis, and loss of appetite and attending outpatient and indoor service were included in the study.

The Patients already taking anti-tubercular drugs and cases of extra pulmonary tuberculosis were excluded from the study.

\section{Sample Collection and Processing}

Sputum samples were collected from these cases in sterile leak proof containers as per RNTCP guidelines. ${ }^{9}$ Sample was divided into 2 parts: one part used for GeneXpert and rest was divided for direct microscopy and decontamination and concentration procedure by NALC-NaOH and 5\% Sodium hypochlorite $(\mathrm{NaOCl})$ or bleach method.

Smears prepared by direct method and decontamination method were subjected to Ziehl-Neelsen staining, Kinyoun staining and Auramine-O staining. The decontaminated samples were inoculated on Lowenstein Jensen Medium slopes (LJ Medium).

\section{Interpretation}

Acid fast bacilli (AFB) were seen as red, beaded rods when assessed under oil immersion (X 100) lens.

The smears were graded as per RNTCP guidelines for $\mathrm{ZN}$ and Kinyoun staining. Whereas for Fluorescent staining the tubercle bacilli appeared bright brilliant greenish yellow against dark background using $40 \mathrm{X}$ lens using Fluorescent microscope and graded as per RNTCP grading for Fluorescent microscopy using Auramine-O stain Reporting scale. ${ }^{10}$ 


\section{Gene Xpert}

GeneXpert assay was put as per Manufacturer's instructions (Cepheid Inc.) and cartridge was loaded into the Gene Xpert Module. The results were read after 2 hours and interpreted as MTB Detected/ MTB Not detected and RIF resistance detected/not detected. ${ }^{11}$

\section{Results and Discussion}

Out of 150 patients, maximum number of the cases was in the age group 21-40 years followed by 41-60 years with mean age being 41.17 years and Male to female ratio was 1.2:1. Most common complaints by the patients were cough (100\%), fever (80\%), dyspnoea (62\%), followed by night sweats (42\%), generalized weakness $(36 \%)$, weight loss (30\%), hemoptysis (24\%) and headache (10\%).

By ZN staining method, 38 (25.33\%) cases were positive for AFB and $112(74.67 \%)$ cases were negative before decontamination i.e. direct microscopy. After decontamination by NALC-NaOH method43 $(28.67 \%)$ cases were positive and $107(71.33 \%)$ cases were negative for AFB. By Bleach processing it detected 39 (26\%) positive cases and 111 (74\%) negative cases. (Table 1)

With Kinyoun Staining, 32(21.33\%) cases were positive for AFB and $118(78.67 \%)$ cases were negative for AFB before decontamination. $35(23.33 \%)$ cases were positive for AFB and 115 (76.67\%) cases were negative for AFB after decontamination by NALC-NaOH method. After bleach processing $32(21.33 \%)$ positive cases and $118(78.67 \%)$ negative cases were detected (Table 1).

By Fluorescent staining using Auramine-O, 40 (26.67\%) cases were positive for $\mathrm{AFB}$ and
$110(73.33 \%)$ cases were negative for AFB before decontamination. After decontamination by NALC-NaOH method 44 $(29.33 \%)$ cases were positive and 106 $(70.67 \%)$ cases were negative for AFB. After Bleach processing $41(27.33 \%)$ positive cases and $109(72.67 \%)$ negative cases were detected (Table 1).

Among 150 samples, only 24 (16\%) sputum samples were found to be culture positive and $85(56.67 \%)$ were negative and 41 (27.33\%) showed contamination on LJ culture.

The total number of positive cases detected by GeneXpert MTB/RIF were 46 (30.67\%) and $104(69.33 \%)$ were negative by GeneXpert MTB/RIF. Out of total 46 positive cases detected by GeneXpert MTB/RIF, 7(15.22\%) cases were found to be resistant to Rifampicin. All the remaining positive cases were found to be sensitive to Rifampicin drug. (Table 2)

GeneXpert MTB/RIF system showed maximum sensitivity and specificity (100\%), followed by Auramine-O which had $86.96 \%$ sensitivity and $100 \%$ specificity. ZiehlNeelsen staining showed $82.61 \%$ sensitivity and $100 \%$ specificity followed by Kinyoun staining which was $69.57 \%$ sensitive and $100 \%$ specific (Table 3 ).

Tuberculosis is an infectious disease which still remains to be a foremost public health risk worldwide. One fourth of the global incident TB cases occur in India annually. Even though there is an availability of many highly sensitive diagnostic tools, early case detection plays a significant role in control of the disease which relies specially on the detection of acid-fast bacilli in clinical samples by microscopy. 12 For early diagnostics and management of TB, various Microscopy methods are available using Ziehl Neelsen, Kinyoun and Fluorescent staining methods. 
Table.1 Sputum smear microscopy findings after various Staining methods

\begin{tabular}{|c|c|c|c|}
\hline & Direct microscopy & After NALC-NaOH & After Bleach \\
\hline ZN Staining & 38 & 43 & 39 \\
\hline Kinyoun Staining & 32 & 35 & 32 \\
\hline Fluorescent Staining & 40 & 44 & 41 \\
\hline
\end{tabular}

Table. 2 Case detection by three different staining methods and GeneXpert

\begin{tabular}{|c|c|c|c|c|}
\hline & ZN Staining & $\begin{array}{c}\text { Kinyoun } \\
\text { Staining }\end{array}$ & $\begin{array}{c}\text { Fluorescent } \\
\text { Staining }\end{array}$ & GeneXpert \\
\hline Positive & $43(28.67 \%)$ & $35(23.33 \%)$ & $44(29.33 \%)$ & $46(30.67 \%)$ \\
\hline Negative & $107(71.33 \%)$ & $115(76.67 \%)$ & $106(70.67 \%)$ & $104(69.33 \%)$ \\
\hline
\end{tabular}

Table.3 Comparison of various staining techniques with GeneXpert

\begin{tabular}{|c|c|c|c|c|}
\hline Diagnostic techniques & $\begin{array}{c}\text { Sensitivity } \\
(\boldsymbol{\%})\end{array}$ & $\begin{array}{c}\text { Specificity } \\
(\boldsymbol{\%})\end{array}$ & $\begin{array}{c}\text { Positive } \\
\text { Predictive } \\
\text { Value (\%) }\end{array}$ & $\begin{array}{c}\text { Negative } \\
\text { Predictive } \\
\text { Value (\%) }\end{array}$ \\
\hline Ziehl-Neelsen Staining & $82.61 \%$ & $100 \%$ & $100 \%$ & $92.86 \%$ \\
\hline Kinyoun Staining & $69.57 \%$ & $100 \%$ & $100 \%$ & $88.14 \%$ \\
\hline Fluorescent staining & $86.96 \%$ & $100 \%$ & $100 \%$ & $94.55 \%$ \\
\hline GeneXpert & $100 \%$ & $100 \%$ & $100 \%$ & $100 \%$ \\
\hline
\end{tabular}

To improve turn around time, Cartridge based Nucleic Acid Amplification techniques i.e. CBNAAT also known as GeneXpert MTB/RIF are nowadays preferred choice due to its rapid results, high sensitivity and no technical expertise.

In the present study on comparing the three different staining methods, detection rate of acid fast bacilli (AFB) by prior decontamination was more with Fluorescent (29.33\%), followed by Ziehl Neelson (ZN) $(28.67 \%)$ and then by Kinyoun staining (23.33\%).Studies conducted by Lawrence et al., and Purusothaman $\mathrm{K}$ et al., were also in concordance with present study. ${ }^{10,13}$ Another study by Saroj et al., demonstrated the superiority of Auramine-O staining over Ziehl-Neelsen staining. ${ }^{4}$ Though Fluorescent microscopy is more sensitive than ZiehlNeelsen staining, but real disadvantage of flourochrome method is that fluorescence fades with time along with cost of microscopy. Therefore slides must be read within 24 hours. Whereas $\mathrm{ZN}$ staining is preferred by most of the countries in the globe, especially in developing countries because of its simplicity and cost efficiency. The only disadvantage of ZN staining is its low utility in HIV- TB coinfected patients and extra pulmonary TB. ${ }^{14}$ Nowadays WHO recommends that conventional fluorescence microscopy be replaced by LED microscopy and that LED microscopy be phased in as an alternative to the conventional $\mathrm{ZN}$ microscopy in all small as well as big laboratories. ${ }^{15}$

Sputum is contaminated with saliva, mucus and normal flora therefore; decontamination concentrates the bacilli and significantly increases the detection rate. ${ }^{9}$ In present study, the detection rate of AFB improved after 
decontamination by NALC-NaOH method more as compared to Bleach method. Similar studies were also reported at Dhaka ${ }^{16}$ and By Kumar et al., which supports present study and hence these are comparable. ${ }^{13}$

Low culture positivity (16\%) in the present study could be explained by complex selection pressure present in the medium that did not allow small number of bacilli in the sputum to grow. ${ }^{17}$ Another reason for low recovery of bacilli could be due to lack of achievement of exact point of neutralization after decontamination. ${ }^{18}$

Staining techniques when compared with Gene Xpert it was found that Gene Xpert is more sensitive and specific (100\%) detecting 2 additional MTB positive cases which were labeled as sputum smear negative. Out of total 46 positive cases detected by GeneXpert $7(15.22 \%)$ cases were found to be resistant to Rifampicin. All the remaining positive cases were found to be sensitive to Rifampicin drug. Amongst staining techniques, Fluorescent staining was more sensitive followed by $\mathrm{ZN}$ staining and Kinyoun staining.

In a study by Aggarwal et al., and Ondimu TO et al., GeneXpert had a higher sensitivity than AFB smear microscopy in respiratory samples similar to our study. ${ }^{19,20}$ In a study by Nakate Prasanna et al., GeneXpert indicated higher sensitivity for detection of TB bacilli as compared to staining methods. ${ }^{21}$ In a study by Dzodanu Eben et al., 35.5\%, 23\%, and 42\% were positive for pulmonary tuberculosis when Fluorescent staining, ZN staining, and XPERT MTB/RIF assay were conducted showing similar trend as present study. ${ }^{22}$

Gene Xpert was closed system test which reduced the chance of contamination of the samples and minimized false interpretations. Positive GeneXpert, but culture negative results should be read cautiously and be well correlated with clinical and treatment history of the patient. The other major advantage of Gene Xpert was that it simultaneously detected Rifampicin resistance and especially beneficial in patient with MDR and HIV associated tuberculosis.

The detection limit of Gene Xpert was 131 bacilli/ $\mathrm{ml}$ which make it more sensitive and reliable test. It could detect small number of bacilli in sputum sample. GeneXpert can be a useful tool for early diagnosis of patients with high clinical suspicion of pulmonary tuberculosis.

Amongst all of the staining techniques studied, Fluorescent staining detected more number of Acid fast bacilli in sputum samples followed by Ziehl-Neelsen staining and Kinyoun staining.

Moreover, the various decontamination methods used increased the detection rate of acid fast bacilli in previously negatively labeled sample hence increasing the diagnostic yield in detecting the bacilli. GeneXpert MTB/RIF had higher sensitivity and specificity as compared to the three staining methods used in our study. GeneXpert test not only detected the $M$. tuberculosis but also detected Rifampicin resistance which was an added advantage over staining methods.

Tuberculosis is a global pandemic. Its early diagnosis and timely initiation of treatment is necessary. The compliance of treatment is also necessary to prevent MDR-TB. In this context, GeneXpert MTB/RIF is a valuable diagnostic test which helps in detecting Tuberculosis in just 2 hours along with Rifampicin resistance. It is also beneficial for pediatric patients and HIV-AIDS patients which usually come out to be sputum smear negative. Therefore, GeneXpert when used in conjunction with microscopy improves detection of tuberculosis and multi drug resistant tuberculosis. 


\section{References}

1. Hymavathi R, Swarnalatha G, Nagajyothi B, Reddy BS. Evaluation of CBNAAT in the diagnosis of smear negative tuberculosis in a tertiary care hospital. Journal of Evolution of Medical and Dental Sciences. 2017 Nov 20;6(90):6273-7.

2. A Global Threat [Internet]. T B Alliance. 2020 [cited 5 September 2020]. Available from: https://www.tballiance.org/whynew-tb-drugs/global-pandemic.

3. T B Statistics India - latest numbers - T B Facts [Internet]. T B Facts. 2020 [cited 17 September 2020]. Available from: https://tbfacts.org/tb-statistics-india/.

4. Bhumbla U. A comparative study of Ziehl-nelson staining and auramine staining in sputum sample for the diagnosis of pulmonary tuberculosis. International Journal of Biomedical Research. 2014;5(6):383-5.

5. Sastry A, Sandhya B. Essentials of Medical Microbiology. 2nd ed. Delhi: Jaypee Brothers Medical Publishers; 2018.

6. Kumar G A, Chandrasekaran S, Visalasree J, Kanna B V. Bleach method in comparison with NALC-NaOH specimen processing method for the detection of mycobacterium in sputum specimen. International Journal of Research in Medical Sciences.2017;5:3865-3868.

7. World Health Organization. Automated real-time nucleic acid amplification technology for rapid and simultaneous detection of tuberculosis and rifampicin resistance: Xpert MTB. World Health Organization; 2013.

8. Boehme C C, Nicol M P, Nabeta P, Michael J S, Gotuzzo E, Tahirli R, Gler M T, Blakemore R, Worodria W, Gray C, Huang L. Feasibility, diagnostic accuracy, and effectiveness of decentralised use of the Xpert MTB/RIF test for diagnosis of tuberculosis and multidrug resistance: a multicentre implementation study. The lancet. 2011 Apr 30;377(9776):1495-505.

9. Revised National T B Control Programme Training Manual for Mycobacterium tuberculosis Culture and Drug susceptibility testing; 2009.

10. Lawrence, Debbarma Madhumita, Baveja C. P., Kumar Surinder, Khanna Ashwani, Sapriina Joseph. Comparative evaluation of fluorescent staining with ziehl-neelsen and kinyoun staining in the diagnosis of clinically suspected cases of pulmonary tuberculosis. Int J Contemp Med Res 2016;3(7):1970-1974.

11. Lawn S D, Nicol M P. Xpert® MTB/RIF assay: development, evaluation and implementation of a new rapid molecular diagnostic for tuberculosis and rifampicin resistance. Future microbiology. 2011 Sep;6(9):1067-82.

12. Amar Kumar G, Chandrasekaran S, Visalasree J, Vignesh Kanna B. Bleach method in comparison with NALC-NaOH specimen processing method for the detection of mycobacterium in sputum specimen. International Journal of Research in Medical Sciences. 2017 Sep;5(9):3865.

13. Purusothaman K, Bhattacharjee K, Joshi $S$, Vasanthakumari R. Comparative efficacies of three acid-fast staining techniques under field conditions for Mycobacterium tuberculosis in the Indian context. The Internet Journal of Microbiology. 2009;9:49-51.

14. Fluorescent light- emitting diode (LED) microscopy for the diagnosis of pulmonary tuberculosis. WHO, Geneva, Switzerland. 2010.

15. Report of the 9th meeting of STAG-TB, Geneva, Switzerland. World Health Organization, Geneva, Switzerland, 2007.

16. Menon S, Dharmshale S, Chande C, Gohil A, Lilani S, Mohammad S, et al., 
Drug resistance profiles of Mycobacterium tuberculosis isolates to first line anti-tuberculous drugs: A five years study. Lung India.2012;29:227-231.

17. Mechal Y, Benaissa E, Benlahlou Y, Bssaibis F, Zegmout A, Chadli M, Malik Y S, Touil N, Abid A, Maleb A, Elouennass M. Evaluation of GeneXpert MTB/RIF system performances in the diagnosis of extrapulmonary tuberculosis. BMC Infectious Diseases. 2019 Dec;19(1):1-8.

18. Kassaza K, Orikiriza P, Llosa A, Bazira J, Nyehangane D, Page A L, Boum Y. Lowenstein-Jensen selective medium for reducing contamination in Mycobacterium tuberculosis culture. Journal of clinical microbiology. 2014 Jul 1;52(7):2671-3.

19. Agrawal M, Bajaj A, Bhatia V, Dutt S. Comparative study of GeneXpert with ZN stain and culture in samples of suspected pulmonary tuberculosis. Journal of clinical and diagnostic research: JCDR. 2016 May;10(5):DC09.
20. Ondimu T O, Grace M O, Samson A O, Asito S A. Comparative study of smear microscopy, GeneXpert and culture and sensitivity assays in detection of mycobacterium tuberculosis on sputum samples among tuberculosis suspected cases in Nyamira County referral hospital. Mycobacterial diseases. 2017;7.

21. Nakate P, Patil S, Patil S, Purohit H, Shelke Y, Kinare S. Comparison of diagnostic efficacy of GeneXpert MTB/RIF assay with Ziehl Neelsen staining \& microscopy in diagnosis of pulmonary tuberculosis. International Journal of Medical Microbiology and Tropical diseases.2019;5(4):218-221.

22. Dzodanu E G, Afrifa J, Acheampong D O, Dadzie I. Diagnostic yield of fluorescence and Ziehl-Neelsen staining techniques in the diagnosis of pulmonary tuberculosis: A comparative study in a district health facility. Tuberculosis research and treatment. 2019 Apr $10 ; 2019$.

\section{How to cite this article:}

Loveena Oberoi, Naveen Pandhi, Muskan Khullar and Tavishi Oberoi. 2021. Comparative Evaluation of Gene Xpert and Various Staining Techniques in the Diagnosis of Pulmonary Tuberculosis. Int.J.Curr.Microbiol.App.Sci. 10(06): 532-538. doi: https://doi.org/10.20546/ijcmas.2021.1006.058 